\title{
Sensor Network Based Localization for Continuous Tracking Applications
}

\author{
[Invited Paper] \\ Livio Denegri
denegri@dist.unige.it \\ Sandro Zappatore \\ sandro.zappatore@unige.it franco@dist.unige.it \\ Department of Communication Computer and System Sciences, DIST \\ University of Genoa \\ 13 Via all'Opera Pia \\ 16145 Genoa, Italy
}

\begin{abstract}
The increasing interest in systems able to provide users immersive services (e.g. domotics, context-aware applications, immersive distance learning tools) has encouraged the development of cheap and effective platforms aimed at tracking objects and people within a certain space. In this context, wireless sensor networks (WSNs) can play a very important role, since specialized sensors can be fruitfully exploited in order to generate/receive signals by means of which the WSN can derive the position of nodes jointed to the objects to be tracked.
\end{abstract}

The paper presents an original localization platform that exploits a single-hop WSN, based on a Microchip MCU and a Cypress RF device, to tracking its moving nodes. Specifically, the nodes of the network are divided in three sets: the first set consists of anchor nodes that, accordingly to the commands from the sink (the central node of WSN), generate ultrasonic pulses. These ones are received by the second set of (moving) nodes, which estimate the pulse-triptime and communicate it to the sink. Finally, the last set is constituted by general purpose nodes that collect any kind of data from the surrounding field. The sink gathers all the data, computes the position of moving nodes and transfers information to external users on the Internet.

The algorithms adopted to manage the network and to localize moving nodes are discussed. A working prototype based upon the hardware platform, software and protocol described in this paper has been deployed and tested, and some results are shown.

\section{Categories and Subject Descriptors}

C.2.1 [Computer Systems Organization]: ComputerCommunication Networks-wireless communication

\footnotetext{
Permission to make digital or hard copies of all or part of this work for personal or classroom use is granted without fee provided that copies are not made or distributed for profit or commercial advantage and that copies bear this notice and the full citation on the first page. To copy otherwise, to republish, to post on servers or to redistribute to lists, requires prior specific permission and/or a fee.

IMMERSCOM 2007, October 10-12, Verona, Italy

Copyright (C) 2007 978-963-9799-06-6

DOI 10.4108/ICST.IMMERSCOM2007.2080
}

\section{General Terms}

Design, Measurements, Performance

\section{Keywords}

Sensor networks, localization, ultrasonic

\section{INTRODUCTION}

Nowadays, more and more systems have localization as the key element to speed up work or to provide advanced services to users: in the so called context aware systems, for example, to have knowledge of the position is the base feature around which all services are developed.

Moreover, localization functionalities are a fundamental component in immersive communications, at last in all situations involving user mobility. As pointed out, e.g., in [4], there are four common tasks in immersive visualization, namely, localization, orientation, navigation and representation. Actually, many works on virtual immersive environments focus on the task of having a user localize a specific target (e.g., an audio source) $[1,4,5]$. However, equally important in such environments is often the symmetric problem of localization of the user on the part of the surrounding ambient technology (see, e.g., $[7,13]$ )

The problem of localization has been commonly solved by using different approaches, which rely upon the environment where the system has to operate. When the application is deployed in a wide outdoor environment, GPS is the most ordinary approach: it works well when receivers are in wide areas, but it can be useless in a more complex environment, such as city narrow streets or indoor spaces [3].

Other solutions have been developed to overcome these deficiencies: radio power maps and theoretical, or empirical, power-decrease laws are often used to estimate the distance from known fixed positions, for example by measuring received power from 802.11 access points or cellular base stations. For indoor applications, also the approaches based on received power do not provide enough accuracy, owing to environmental complexity. Improvements are obtained when other techniques are used: solutions based on ultrasonics give better results than radio approaches and they are cheaper solutions, too. 
When high precision is the goal, systems are usually based on high technological devices or very complex infrastructures, which make the deployment expensive and difficult, especially indoor. Wiring takes most of the effort: solutions based on wireless communications can easily overcome this trouble and therefore can speed up system deployment.

Wireless Sensor Networks (WSNs) embody the idea of flexibility and easiness. They are composed of several simple devices, the nodes, which communicate by radio and cooperate to reach a goal. WSNs are commonly used to monitor very wide areas: nodes measure some quantity, for example temperature, and send data toward a specific node, called sink, which is the interface between the WSN and other networks. Specific protocols have to be developed to configure the network, by flooding topological information, and to route data from sensors to the sink. To overcome long distances, protocols must allow multihop transmissions. Nodes send packets to the sink by means of other nodes, but they must also be as simple as possible to save batteries and due to limited computational and storage capabilities of the nodes. WSNs can be organized as meshes, by exploiting multihop protocols, but also clusters or hierarchical structures can be adopted during network design.

Localization, in an indoor environment, can be well faced by using WSNs: a subset of the nodes forms the fixed infrastructure, while the others are attached to what has to be localized. The availability of cheap single chip computers and miniaturized radio-transceivers makes easy to design small dimension nodes, which can be installed everywhere without many difficulties.

The paper is organized as follows. Section 2 describes the structure of the WSN, the nodes and the algorithms implemented. Measurements done on the communication channel and localization precision results are shown in Section 3. Finally, in the last section, conclusions are drawn and future improvements to the system designed are proposed.

\section{THE SENSOR NETWORK}

Figure 1 sketches the devised sensor network, whose peripheral elements, the nodes, are the components of a platform devoted to the tracking application. Nodes can be divided in three main sets, according to the role they play within the network. The first set consists of the nodes, called Anchor Nodes (AN), that must placed in fixed, suitable (and known) positions; the second set is represented by the moving nodes, here referred as Mobile Nodes (MN), which have to be tracked within a certain building or room. Eventually, the last set groups general purpose nodes (GN), whose goal might be, for instance, the monitoring of some environmental parameter, as temperature. All these nodes directly communicate, via a Radio Modem (RM), with a central node, called sink, which gathers the data acquired by the peripheral nodes, synchronizes network operations, and acts as a "gateway" to an IP infrastructure.

It should be highlighted that, in our implementation, the hardware platform has been designed with off-the-shelf elements: this allows building very cheap nodes, characterized by good computational and communication capabilities. To this aim, during the design phase, much attention was paid

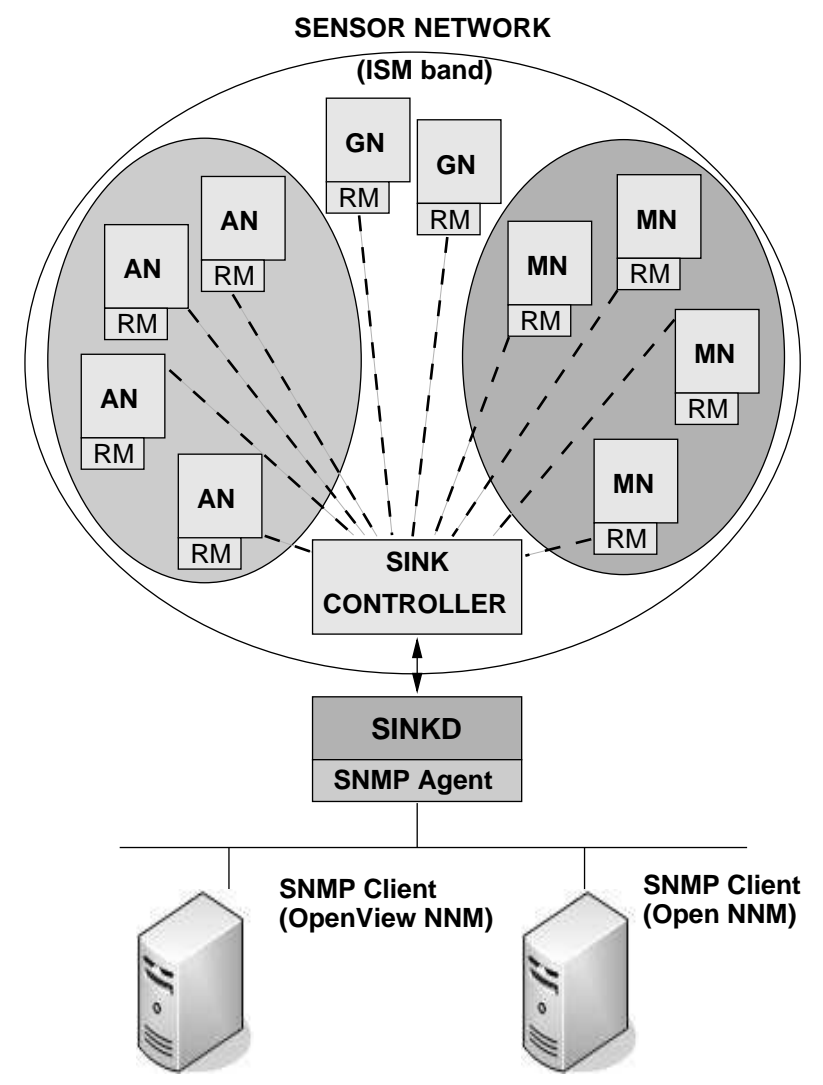

Figure 1: Overall structure of the proposed sensor network, publishing data by means of a SNMP agent.

in order to i) adopt commercial components, ii) choose integrated circuits providing a PDIP (Plastic Dual Inline Package) package (in this manner any possible part replacement is quite simple), iii) to exploit a RF transceiver operating in the free ISM band $(2.4 \mathrm{GHz})$, able to internally spread and serialize/de-serialize data to be transmitted, and iv) keep the size of the node board as small as possible, thus permitting an easy deployment of the WSN.

The Anchor, Mobile and General purpose nodes are based upon the same hardware platform, consisting of a main board, on which all the electronic components are placed. The different "identities" of the nodes are provided by the specific daughter card plugged into the main board. At the moment two daughter cards have been designed: one, used in the ANs, includes an amplifier and an ultrasonic emission device [9]. The other, employed in the MNs, includes an ultrasonic microphone [9], and a band pass front-end amplifier. The GNs require no daughter cards, at least for the most common data acquisitions.

The functional blocks of a main board are depicted in Fig. 2. Its core component is represented by the MCU, a PIC $16 \mathrm{f} 876$ [12] (or PIC 18f252, that is pin-to-pin compatible with the previous one): the latter communicates, via a SPI (Serial Peripheral Interface), with a radio transceiver, based on the Cypress CYWUSB6935 chip [2]. In our implementation, we adopted the Cypress based "High speed multi-channel 


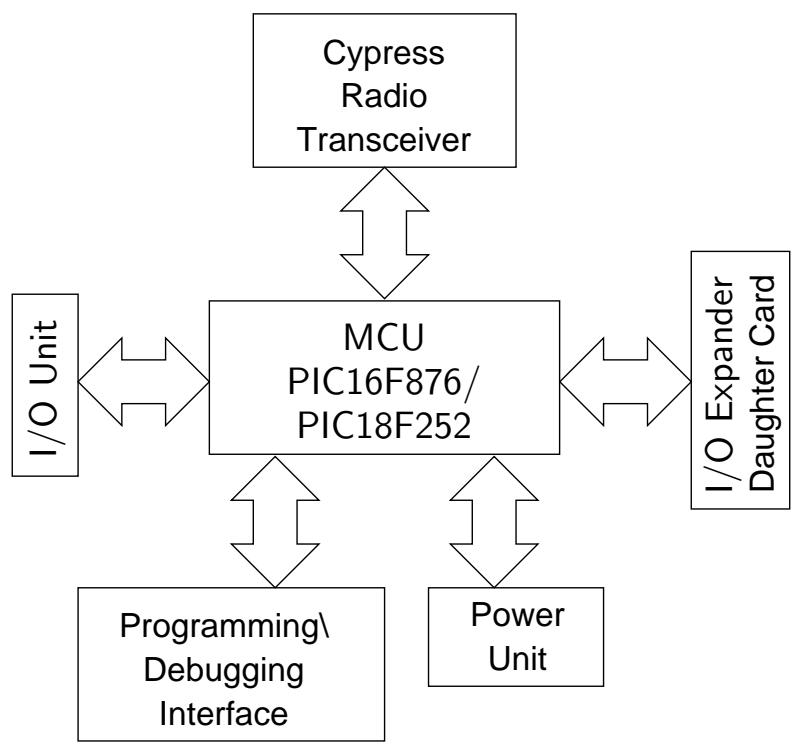

Figure 2: Functional blocks of a WSN node.

transceiver", produced by Aurel S.p.A., an Italian medium enterprise, specialized in RF modules design [10].

All the analog inputs of the MCU, SPI and PWM (Pulse Width Modulator) lines and other MCU general purpose signals are available at the connector, which a daughter card can be plugged in. In this manner, it is possible to control and acquire a greater number of signals, as well as to handle special purpose cards, consisting of an ad-hoc electronic circuitry, as in the case of the MNs and ANs.

The Programming/Debugging interface provides an ICD (InCircuit Debugging) tap, which allows the user to upload and debug the firmware directly on the MCU. The Power Unit (PU) monitors the battery status and, if an external power supply is available, the PU controls and regulates the battery charge. The I/O unit is represented by a very simple interface, which permits the MCU to acquire only a very limited number of signals from the field. Although the MCU can handle 8 digital (viz. on-off) inputs, 8 digital outputs and 4 analog input channels, the on-board circuitry permits to manage only 1 digital input, 1 digital output, and 1 analog input channel: to achieve more I/O capacity a daughter card must be plugged in the main board.

This design choice is motivated by the fact that, in general, it is convenient to uncouple the network operation facilities and functionalities, which reside on the main board of a node, from specific capabilities that involve the use of adhoc components. Furthermore, it should be noted that, in the case of a GN, only a limited number of I/O lines are generally needed to meet data acquisition requirements.

Figure 3 presents the main board of a sensor developed within our research activity. The various protocol layers of the WSN, as well as the driver handling the RF transceiver, the software portions managing the power unit and the signal acquisition and signal conditioning are implemented on the MCU.

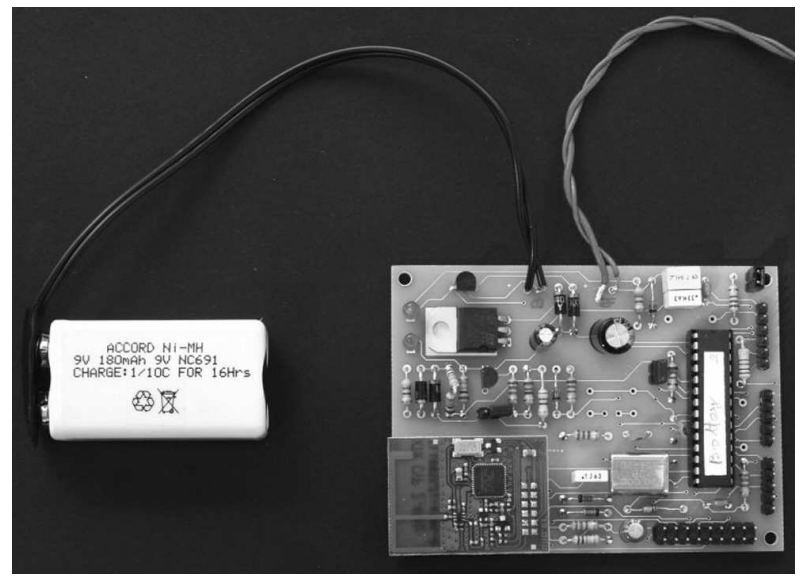

Figure 3: Main board of the implemented wireless sensor

As already mentioned, the central element of the network is the sink, which includes two main elements: a network sink controller (NSC), and a sink manager (SM).

The hardware and firmware of the former are similar to those of a common sensor node, while the latter consists of a single board ARM (Advanced RISC Machine) computer (booting Linux), produced by Technologic Systems [11].

The NSC actually manages the sensor network and provides a proper synchronization, thus permitting the MNs to estimate their distance from the ANs (see Section 2.4). The SM gathers the MNs' data related to the distances (from ANs), and processes them according to a triangulation algorithm. Finally, the SM publishes MNs' positions, by exploiting the facilities offered by a SNMP agent or by a WEB service.

To this aim, the SM continuously communicates with the NSC in order to get any information acquired from the MNs, and to send commands to the ANs, so that they are triggered to generate an ultrasonic pulse. Moreover, the SM collects data acquired from the GNs. The SM stores information from the nodes in an internal real-time database, which is also accessed by the processes involved in the triangulation procedure and in the publishing of data gathered by the GNs. The functional blocks of the sink are depicted in Fig. 4.

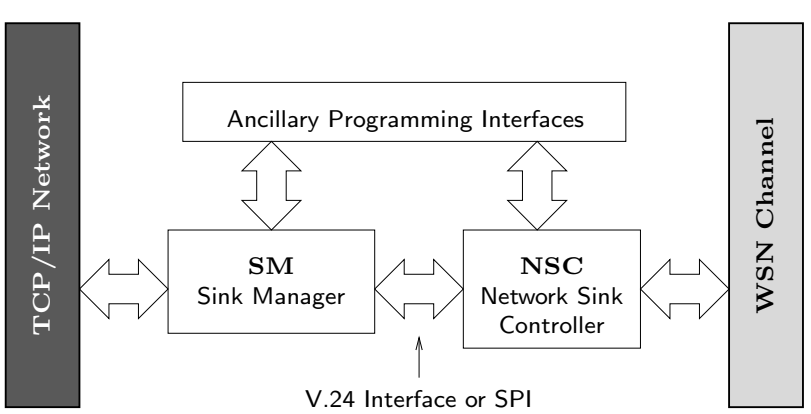

Figure 4: Functional blocks of the sink. 


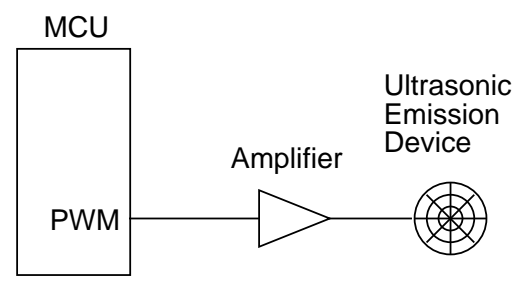

Figure 5: Functional blocks of the ultrasonic transmitter.

\subsection{Anchor node}

In Figure 5 blocks forming the ultrasonic transmission electronics are depicted. As can be seen, the design has been done as simple sa possible to reduce power consumption and dimension. All operations are controlled by the MCU of the main board. Ultrasonic pulses, at the frequency of 40 $\mathrm{kHz}$, are generated by means of the PWM (Pulse Width Modulation) signal produced by the PIC which drives an amplifier/voltage buffer connected directly to the ultrasonic emission device [9].

\subsection{Mobile node}

Mobile nodes ultrasonic electronics is composed by blocks shown in Fig. 6. As for Anchor Nodes pulses detection is performed as simply as possible.

Ultrasonic received signal is first filtered with a Band Pass Filter (BPF) at $40 \mathrm{kHz}$ and then amplified 1000 times to get voltages of about 2 volts. Pulse detection is performed by exploiting MCU external interrupt (INT) facility. When received signal exceeds a threshold the interrupt is generated and the delay of the pulse is measured (see Figure 7). The threshold is generated by filtering the PWM signal provided by the MCU: by varying the duty cycle of the PWM, mean value extracted by the LPF (Low Pass Filter) can be changed. The threshold is determined before a MN begins its operations: its value is chosen above the maximum noise level during a listening window. Proceeding this way delay measurements errors are minimized and the system can adapt to different noise levels.

\subsection{Communication protocol}

The access protocol implemented is quite simple, and can be considered a modified version of a polling algorithm. The basic idea divides the network operation in two different phases. During the former one, called Polling Phase (PP),

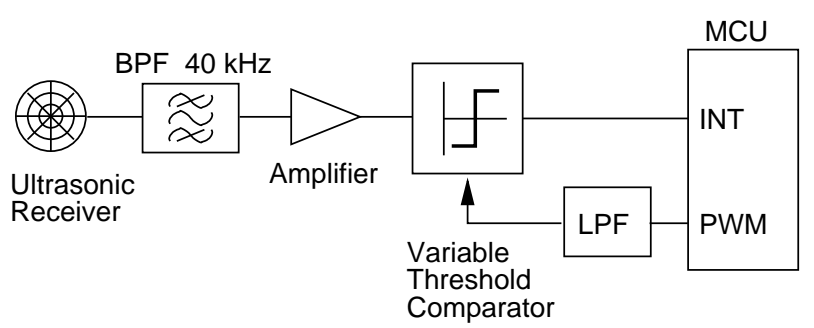

Figure 6: Functional blocks of the ultrasonic receiver.

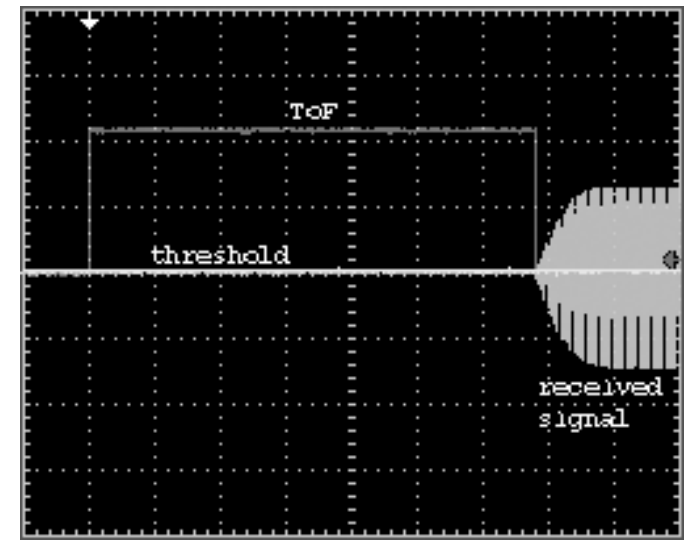

Figure 7: Screenshot showing the received signal, threshold level and the measured ToF.

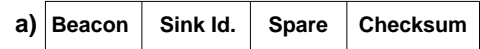

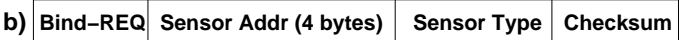

c) Bind-RES \begin{tabular}{|l|l|l|l|l|}
\hline Sensor Addr (4 bytes) & $\begin{array}{l}\text { Assigned } \\
\text { Sensor ld. }\end{array}$ & Poll Time & Spare & Checksum \\
\hline
\end{tabular}

d) \begin{tabular}{r|l|l|l|l|}
\hline Poll-REQ & Sensor Id. & Link Quality & Payload (3 bytes) & Checksum \\
\hline
\end{tabular}

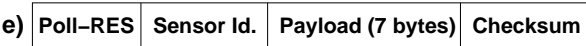

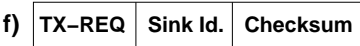

Figure 9: Packet types used within the WSN.

the sink cyclically sends to ANs the command to force the emission an ultrasonic pulse train. Then, the sink polls the $\mathrm{MNs}$, in order to receive, from each $\mathrm{MN}$, the time spent by the pulse to reach it. In order to send/receive commands/data to an AN/MN, the sink transmits a POLLREQuest to an addressed Anchor/Mobile node, which in turn, must respond with a POLL-RESponse packet within a certain time interval.

After sending the commands to all the ANs and polling every MNs, the second phase, named Aloha Phase (AP), starts. The sink periodically broadcasts a beacon (BEAC) and listens to the channel for a certain amount of time. Upon receiving a beacon, a bound GN node may notify (by means of a TX-REQuest) the sink that it has new data acquired from the field, or an un-bound node may ask to enter the network by sending a BIND-REQuest packet.

In the former case, the sink polls the GN, thus enabling it to transmit the information acquired; in the latter case, the sink binds the new node and sends it a Bind-RESponse packet, containing a unique identifier, by means of which the sink will poll the sensor in all the following data exchanges. Figure 8 diagrammatically represents different transaction types between sink and sensor nodes.

The various packet types used within the WSN are reported in Fig. 9. 


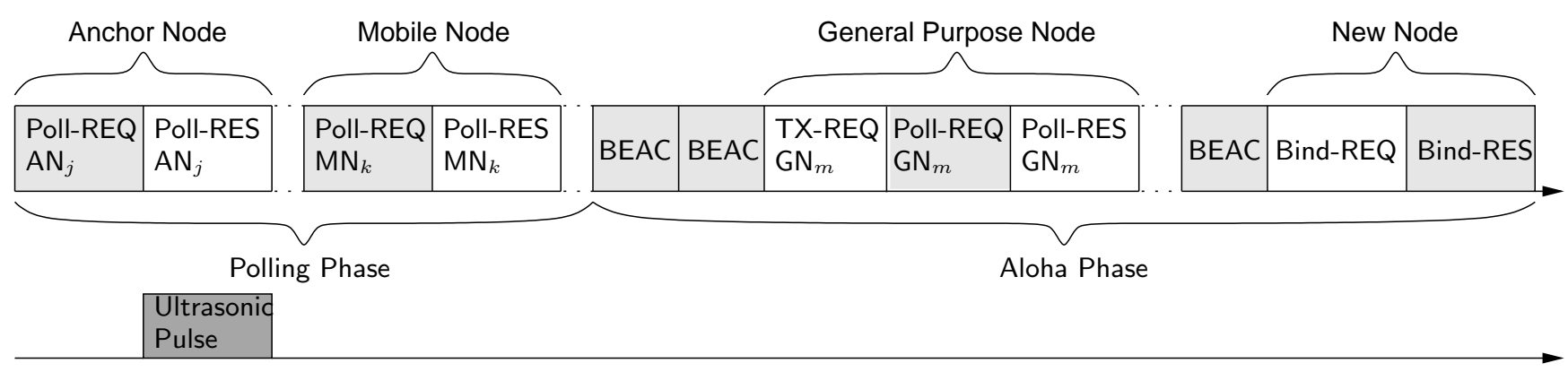

Figure 8: Time line showing: $j$-th AN and $k$-th MN polling (Polling Phase); $m$-th GN request to send and new node binding (Aloha Phase). Packets transmitted by the sink are shown in light grey, white packets are transmitted by nodes and ultrasonic pulse is shown in dark grey.

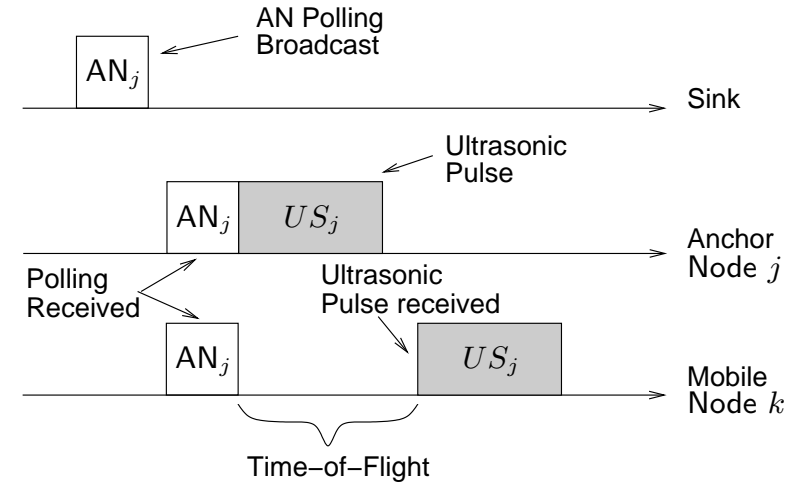

Figure 10: Polling packet sent by the sink to the $j$-th AN and used by the $k$-th MN as a reference time to calculate ToF.

\subsection{Synchronization, measure and data gath- ering}

When the network is set up, mobile nodes' localization can start: Fig. 8 shows the operations taking place. The sink node begins by broadcasting a packet, commanding anchor node $j$ to send an ultrasonic pulse. Because this packet is broadcast, also mobile nodes receive it: so they use this packet as a time reference to measure Time-of-Flight (ToF) of the next ultrasonic pulse. ToF measurement of each mobile node ends when it detects the ultrasonic pulse, as shown in Fig. 10, or after a hard-coded timeout. Ultrasonic detection is performed by using the hardware described in Section 2.2. Since radio propagation delays can be accounted as zero due to the short node-to-node distances of a singlehop network, only firmware and hardware delays have to be canceled out from the ToF to get a precise distance measurement. These delays are caused by radio packet processing and electronics governing ultrasonic generation [8].

A fixed time after command transmission, the sink begins the polling phase to collect measurements from every mobile node. Each polled node transmits its own ToF measurement to the sink, the latter records the measurement: when a mobile node has a set made up of at least three measurements, its position can be fixed. The algorithm used to estimate mobile node position is based on trilateration and will described in Section 2.5.
The polling phase is the most time consuming one, especially if a great number of mobile nodes have to be localized, because all mobile nodes' measurements have to be gathered. To improve this operation, a position-based approach has been introduced. When the system starts localization, the polling phase is executed by polling all the nodes associated with a sink in an impartial way. However, when nodes positions are known, polling can be done by using a more clever approach. Mobile nodes can only detect ultrasonic pulses coming from anchor nodes placed in a small area above them, so when sink commands anchor $\mathrm{AN}_{j}$ to transmit the ultrasonic pulse, it then polls only mobile nodes which were before localized near $\mathrm{AN}_{j}$. Adopting this technique, which works under the hypothesis of slow movements, only few nodes are polled: therefore the duration of the polling phase is reduced.

\subsection{Position fixing}

When at least three Time-of-Flight measurements of the same mobile node are collected, a trilateration algorithm can be used to estimate node position [6].

Assuming the speed of sound equal to $344 \mathrm{~m} / \mathrm{s}$, distances of the mobile node from the anchor nodes are calculated. Heights from the ground are considered fixed, i.e. each mobile node's height is known by the system and does not change during time. This hypothesis is easily fulfilled, for example, when mobile nodes are attached to racks, trolleys or forklift trucks: during the network start up mobile nodes can transmit their heights to the sink. Further improvements to the implemented algorithm will allow to remove this hypothesis.

The first step of the algorithm is to calculate the intersection points of the three Time-of-Flight-radius spheres: by projecting everything onto the plane where the mobile node lies (the height from the ground of anchor nodes and of the specific mobile node is used now) we will consider circles instead of spheres. The radius of a circle is tightly related to Time-of-Flight-radius, so we will use the same notation. Due to errors or inaccuracies during ToF calculation, this first step can give rise to three cases: i) all the circles intersect, so there are six intersection points, ii) not all the circles intersect, so there are four intersection points, and iii) only two circles intersect. 


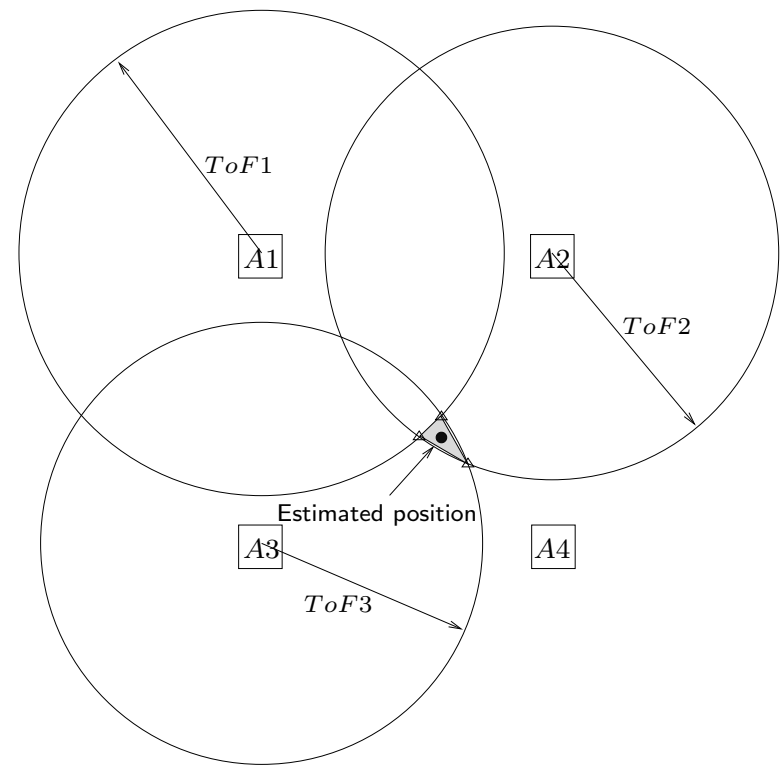

Figure 11: Example of trilateration by using three anchor nodes $(A N j)$. Position is fixed (black circle) in the center of mass of the triangle determined by the intersection points (triangles) of the Time-ofFlight-radius $(T o F j)$ circles.

In the latter case localization is not possible, while in the first two cases, intersections points are processed in the same way during step two of the algorithm. First, the set of the three or two closest points belonging to different intersections is created. Second, the estimated position is evaluated as the center of mass of the three or two points. Figure 11 exactly shows the result when all the Time-of-Flight-radius spheres intersect. The grey triangle is created by the three closest points of the intersecting circles (marked with triangles) and the estimated position is its center of mass (black dot).

\section{EXPERIMENT AND RESULTS}

The test activity had two main objectives. The first one aims at evaluating the actual characteristics of the radiomodems adopted for our WSN. Specifically, the goal was to better characterize the coverage area and interference immunity offered by radio devices under different operative conditions. The second group of test was carried out in order to estimated the accuracy in determining MNs' position achievable with the adopted localization algorithm. As concerns the coverage area and interference immunity, a number of tests were performed on a floor of a building, with several offices and laboratories.

To estimate the coverage area of the radio-transceiver, a set of measurements has been collected on a floor of the building housing our department. The department is formed by two rows of contiguous offices and laboratories, separated by a passageway. In particular, walls between rooms are built with reinforced concrete, while walls between the passageway and rooms are glass panels: it can be considered as a quasi open-space environment. Sink was placed in the middle point of the passageway.

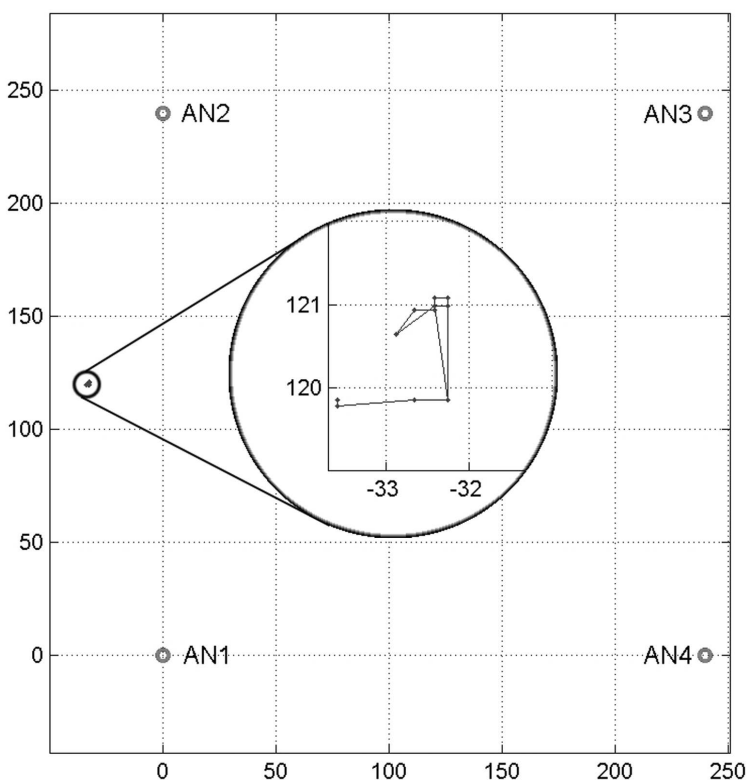

Figure 12: Localization of a still MN. Zoom shows that estimated positions are concentrated in an almost one square centimetre area. All measures are expressed in centimetres.

When packets are transmitted by using the maximum power (15 dBm), coverage area extends to about 25 metres in every directions from the node, that allows to cover the entire floor using a single sink. It has been measured that $95 \%$ of the floor area is covered also by transmitting at $6 \mathrm{dBm}$, that allows to save batteries. Furthermore, long distance measurements, performed under a quasi free space condition, highlighted a maximum transmission range of about 500 metres. This proves that, within an open space (e.g., a storeroom, a hangar), a node may be about 150-200 meters away from the sink: therefore, as long as the sink is suitable placed, the WSN may cover an area of about 25000 square metres.

The interference immunity was evaluated by studying the capability of rejecting cochannel and adjacent-channels interferences. The tests were carried out with the help of another radio-transceiver, suitably programmed in order to generate signals at the same frequency or on an adjacent channel. Cochannel tests were performed using different pseudocodes, while during adjacent-channel test, the main and the interfering signal exploit the same pseudo-code. In both the cases, a good interference immunity has been proved.

Finally, to evaluate the performance of the ultrasonic localization sub-system, a reduced testbed has been adopted. Four ANs have been attached to the ceiling of our laboratory and one $\mathrm{MN}$ has been moved inside the area below them. Figure 12 shows the position estimation of a still MN; Figure 13 shows instead the tracking of a MN moving along a path. The position of a motionless node falls in an area of about one square centimetre: this is due to random ToF measurements errors. The tracking of a MN moving along a path shows greater errors, which are however always less than 20 centimetres. 


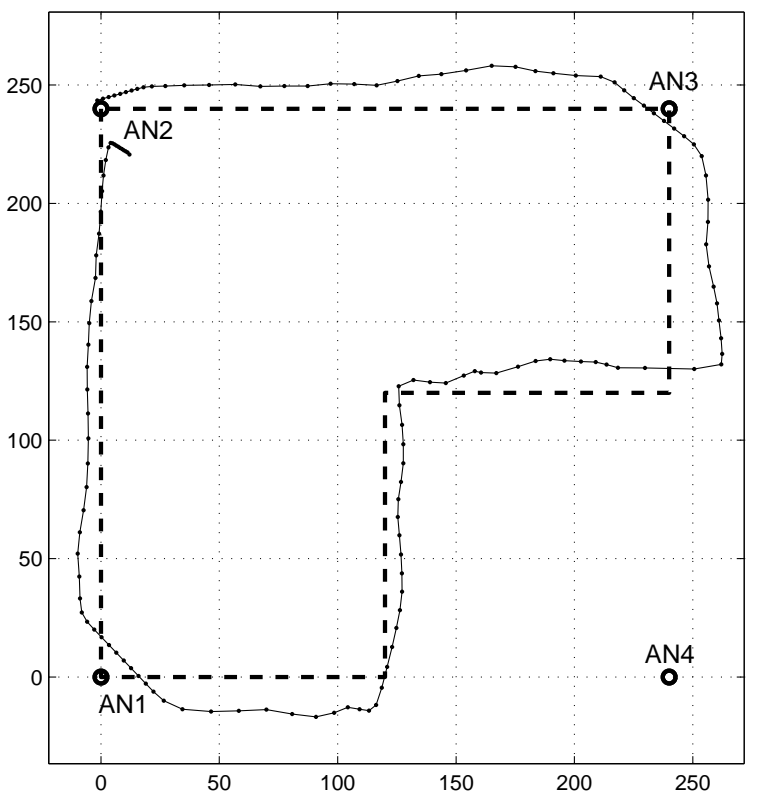

Figure 13: Tracking of a MN (solid line) following a path (dashed line). Maximum error is about 20 centimetres. All measures are expressed in centimetres.

Owing to the narrow beams of the ultrasonic emission devices and receivers, ceiling height and the height of the MNs affect the ultrasonic coverage area of each AN. Specifically, with a ceiling height of about 3 metres, the coverage area of each installed $\mathrm{AN}$ is about a circle of 2.5 metres radius.

\section{CONCLUSIONS}

The paper presented an original WSN, designed and implemented for facing localization needs in an indoor environment. The network operates according to a single hop transmission scheme: a sink coordinates all the anchor nodes and mobile nodes to localize the latter; furthermore, other nodes with different "identities" (for example to measure temperature) are handled by the network.

Measurements have proved that, by using the hardware and the algorithms described here, a sink can control an openspace area of about 25000 square metres; moreover, precision achieved by the ultrasonic localization sub-system is about 2 centimetres for still MNs and 15 centimetres for mobile ones.

Localization precision depends very much on anchor nodes positioning and density in the area of interest (possible damaged nodes must be considered too): future activities will study how to position these nodes to get the best coverage with the less number of nodes. Furthermore, mobile node tracking precision relies on measurements frequency, but also battery life must be take into account: the tradeoff between these two requirements will be analyzed in future activities too.

A possible evolution we are considering consists of using a multi-hop protocol to organize the network in a hierarchical framework. In this way, data coming from sinks are collected by an higher level where nodes act as data concentrators. This approach can be repeated in a pyramidal way to deliver data to a unique control point.

The proposed solution is also suitable for (very) wide WSNs: in this case, the WSN has to be structured in clusters, each of them controlled by a sink. Obviously, a second level network is needed to assure the data exchange among the sinks of the clusters. Furthermore, if the system has to be deployed in environments where ceilings are very high, ultrasonic devices range could be too shortened, thus changes could be necessary in the hardware and/or firmware.

\section{REFERENCES}

[1] J. Breebaart, J. Merre, L. Villemoes, C. Jin, K. Kjörling, J. Plogties, and J. Koppens. Multi-Channel Goes Mobile: MPEG Surrounding Binaural Rendering. In Proc. AES 29th Internat. Conf., Seoul, Korea, September 2006.

[2] Cypress. CYWUSB6935: WirelessUSB TM LS 2.4 GHz DSSS Radio SoC, Datasheet, available on line at http://www.cypress.com.

[3] E. Elnahrawy, L. Xiaoyan, and R. P. Martin. The Limits of Localization Using Signal Strength: a Comparative Study. In IEEE Communications Society Conf. on Sensor and Ad Hoc Communications and Networks, 2004. (SECON04), pages 406-414, October 2004.

[4] M. Gröhn, T. Lokki, and L. Savioja. Using Binaural Hearing for Localization in Multimodal Virtual Environments. In Proc. 17th Internat. Congress on Acoustic, volume 4P, Rome, Italy, September 2001.

[5] M. Gröhn, T. Lokki, L. Savioja, and T. Takala. Some Aspects of Role of Audio in Immersive Visualization. In Proc. of SPIE, pages 13-22, 4302, San Jose, CA, January 2001.

[6] A. Mandal, C. V. Lopes, T. Givargis, A. Haghighat, R. Jurdak, and P. Baldi. Beep: 3D Indoor Positioning Using Audible Sound. In Second IEEE Consumer Communications and Networking Conference, 2005 (CCNC05), pages 348-353, Las Vegas, January 2005.

[7] Y. Nakazato, M. Kanbora, and N. Yokoya. Discrete Markers for User Localization. In Proc. 8th Internat. Symp. on Wearable Computers (ISWC'04), pages 172-173, Arlington, VA, 2004.

[8] N. Priyantha, A. Chakraborty, and H. Balakrishnan. The Cricket Location-Support System. In 6th ACM/IEEE Internat. Conf. on Mobile Computing and Networking, August 2000.

[9] See for further information www.alldatasheet.com/datasheetpdf/pdf/109543/ETC/400ST160.html.

[10] See for further information www.aurelwireless.com.

[11] See for further information www.embeddedarm.com.

[12] See for further information www.microchip.com.

[13] R. Singh, C. S. Regazzoni, and K. Plataniotis. Astatistical Modeling Versus Geometrical Determination Location Approach for Static Positioning in Indoor Environment. In Proc. IEEE WPMC, Aalborg, Denmark, September 2005. 